\title{
Joanna Woźniak (2016): Fachphraseologie am Beispiel der deutschen und der polnischen Fassung des Vertrags von Lissabon, Frankfurt am Main, 307 S. Schriftenreihe: Danziger Beiträge zur Germanistik, Bd. 52. ISBN 978-3-631-67482-6.
}

\author{
DOI: $10.19195 / 0137-1169.37 .8$
}

Die Monografie von Joanna Woźniak „Fachphraseologie am Beispiel der deutschen und der polnischen Fassung des Vertrags von Lissabon“" wurde 2015 im Wettbewerb „Peter Lang Nachwuchspreis“" ausgezeichnet. Die Studie beschreibt und klassifiziert Fachphraseologismen aus dem Vertrag von Lissabon und schließt somit eine Lücke in der Erforschung von Fachphraseologismen aus der deutsch-polnischen kontrastiven Sicht. In ihrer Arbeit widmet sich die Autorin grundlegend drei Forschungsfragen - dem Anteil der Fachphraseologismen im Gesamtwortschatz des untersuchten Gesetztextes, den Klassen und der Beschreibung der Fachphraseologismen sowie ihrem Fachlichkeitsgrad.

Das Buch ist übersichtlich und klar strukturiert und umfasst fünf Kapitel, wobei die ersten drei die theoretische Grundlage bilden. Ausgegangen wird in dem ersten Kapitel von der Beschreibung der Fachsprachen. Hierzu führt die Autorin sowohl deutsche als auch polnische Begriffsbezeichnungen und Definitionen an, die das Wesen der Fachsprachen bestimmen. Die Merkmale der Fachsprachen werden von der Autorin sehr detailliert und schlüssig dargelegt und zwar nach dem Kriterium der sprachlichen Ebene. Auf der Ebene der Lexik zeichnen sich Fachphraseologismen u.a. durch Fachbezogenheit, Exaktheit, Eindeutigkeit, Explizitheit und Begrifflichkeit aus. Die Autorin bemerkt dazu, dass neueste Untersuchungen auch Merkmale der Vagheit und Synonymie des Fachwortschatzes anführen. Zu Recht macht Woźniak darauf aufmerksam, dass sich das Wesen von Fachsprachen sowohl auf der Ebene der Lexik, als auch in der Morphologie und der Syntax manifestiert. So spricht man in diesem Zusammenhang sowohl im Deutschen als auch im Polnischen von dem dominierenden Anteil der Nomina, im Deutschen besonders der Zusammensetzungen. Syntaktisch betrachtet, sind für Fachsprachen Satzkomplexität oder Verwendung von unpersönlichen Konstruktionen ausschlaggebend. Im Vordergrund der Monografie steht die juristische Sprache, die eine besondere Art der Fachsprache darstellt - ,[die] Besonderheit der juristischen Fachsprache liegt [...] in ihrer nahen Verbindung mit dem alltäglichen Leben und daher wird ihr im Unterschied zu anderen Fachsprachen die Forderung nach Allgemeinverständlichkeit gestellt“‘ (S. 40).

Den Gegenstand des zweiten Kapitels bilden textsortenspezifische Merkmale von Fachtexten, dargelegt vorwiegend anhand des Mehrebenenmodells von Heinemann / Heinemann (2002). Nach einleitenden Überlegungen verweist die Autorin auf den Aspekt der Formelhaftigkeit, der aus der Perspektive der Fachsprachen besonders relevant scheint. Formelhaftigkeit und Konventionalität (darunter Formulierungskonventionen) kommen besonders in Rechtstexten zum Tragen. Schon an dieser Stelle wird die Rolle von Phraseologismen 
deutlich. Im Vordergrund stehen dabei pragmatische Phraseologismen (Routineformeln), die situationsabhängig und für bestimmte Textsorten prädestiniert sind. Im Anschluss an diese Überlegungen wird der Vertrag von Lissabon fachtextlinguistisch dargelegt.

Im dritten Kapitel gibt die Autorin einen Überblick über die Fachphraseologie in der Rechtssprache. In Anlehnung an maßgebliche Arbeiten zur allgemeinen Phraseologieforschung wie auch zur Fachphraseologie führt die Autorin Merkmale der untersuchten Phraseologismen auf. Dieser Darstellung folgt eine detaillierte Klassifikation von Fachphraseologismen. Von der Autorin werden in diesem Zusammenhang folgende Klassen unterschieden:

- Phraseologische Termini

- Fachphraseme

- Fachkollokationen

- Funktionsverbgefüge

- Lateinische Phrasen

- Pragmatische Phraseologismen.

Die festgesetzten Klassen bilden die Grundlage für die deutsch-polnische kontrastive Analyse der Fachphraseologismen im Vertrag von Lissabon, die im vierten Kapitel durchgeführt wird. Ein besonderes Augenmerk wird dabei auf die deutschen Fachphraseologismen und ihre polnischen Entsprechungen gerichtet. In ihrer Analyse untersucht die Autorin 1280 feste Syntagmen, die sie dem Vertrag entnommen hat. Die Untersuchung des genannten Materials erlaubt, sowohl quantitative als auch qualitative Schlüsse zu ziehen. Die größte Gruppe in dem untersuchten Bereich bilden Fachkollokationen, gefolgt von phraseologischen Termini und pragmatischen Phraseologismen. Am seltensten werden indessen Fachphraseme verwendet. Die exzerpierten Syntagmen machen insgesamt ca. 20\% des Gesamtwortschatzes beider Verträge aus. Den größten Anteil an Fachkollokationen begründet die Autorin mit dem konventionellen Charakter des untersuchten Textes. Der große Anteil phraseologischer Termini ist ebenfalls dadurch begründet, dass sie die Grundlage aller Fachtexte bilden. Die für die Rechtssprache typischen Routineformeln beweisen indessen die Konventionalität und Formelhaftigkeit juristischer Texte. Funktionsverbgefüge werden im Vertrag seltener verwendet aber mehrmals wiederholt, was ebenfalls auf eine bestimmte Textfixierung verweist. Unter den 1280 Syntagmen lassen sich lediglich zwei Fachphraseme finden. In der Verwendung der Fachphraseologismen in den beiden Sprachen konnte die Autorin sehr viele Similaritäten erforschen. Auf der Ebene der Wortbildung konnte in beiden Sprachen ein großer Anteil von Internationalismen nachgewiesen werden. Der Autorin ist es auch gelungen, die häufigsten Strukturmuster von Fachphraseologismen zu ermitteln - in beiden Sprachen sind es aus einem Nomen und einem Adjektiv bestehende Syntagmen. Des Weiteren konnte Woźniak in ihrer Analyse nachweisen, dass den deutschen Syntagmen meist auch in der polnischen Sprache Syntagmen entsprechen. Zuletzt widmet sich die Autorin in diesem Teil der Monografie dem Fachlichkeitsgrad der extrahierten Phraseologismen. Unter diesem Aspekt erweist sich das Material als uneinheitlich. Die Autorin konnte somit sowohl Phraseologismen mit hochspezifischem als auch mit allgemeinwissenschaftlichem Charakter ermitteln.

Im letzten Kapitel werden Schlussfolgerungen gezogen und ein Ausblick vorgenommen. An dieser Stelle wird auf die Möglichkeit der Verwendung der Studie sowohl in kontrastiven als auch in didaktischen Untersuchungen verwiesen. Ihre Arbeit betrachtet die Autorin auch als einen Einstieg in größer angelegte Studien und Analysen zu Fachphraseologismen. 
In dem die Arbeit abschließenden Satz kommt die Autorin noch einmal darauf zurück, dass ihre Arbeit einen Beitrag zur Erforschung von Fachphraseologismen in der Rechtssprache leisten sollte. Dies ist der Autorin durchaus gelungen. Das Buch ist eine fundierte und detaillierte Studie zu juristischen Fachphraseologismen. Die Studie ist auf soliden theoretischen Grundlagen aufgebaut und bietet eine umfassende Analyse, die weitreichende Schlüsse erlaubt. Diese können sowohl aus der Perspektive der Translatorik als auch der DaF-Didaktik von Nutzen sein. Das Buch „Fachphraseologie am Beispiel der deutschen und der polnischen Fassung des Vertrags von Lissabon“ leistet somit ganz gewiss einen wertvollen und wissenschaftlich relevanten Beitrag zu Untersuchungen im Bereich der Fachphraseologie.

\section{Claire Ellender (2015): Dealing with Difference in Audiovisual Translation. Subtitling Linguistic Variation in Films (New Trends in Translation Studies, vol. 14), Oxford/Bern/Berlin/Bruxelles/Frank- furt a.M./New York/Wien: Peter Lang, 221 S. ISBN 978-3-0343- 1816-7.}

DOI: $10.19195 / 0137-1169.37 .9$

Die zu besprechende Publikation ist in der Serie „New Trends in Translation Studies“ (Band 14) einer internationalen Reihe erschienen, derer Ziel es ist, wissenschaftliche Forschung auf dem Gebiet der Übersetzungs- und Dolmetschwissenschaft zu fördern. Überdies erfüllt sie die Rolle eines Forums für die Gemeinschaft der Translationswissenschaftler.

Die Monografie besteht aus sechs Kapiteln, denen Tabellenverzeichnis, Danksagung und Einleitung vorangeht. Die Verfasserin setzt sich zum Ziel zu untersuchen, vor welche Herausforderungen sich die Untertitler beim audiovisuellen Übersetzen sprachlicher Varietäten des Englischen ins Französische und des Französischen ins Englische gestellt sehen. Außerdem wird erforscht, welche Lösungen für die Übersetzungsprobleme gefunden wurden. Die in den jeweiligen Kapiteln analysierten Aspekte der Sprachvarietäten im audiovisuellen Übersetzen münden in Schlussfolgerungen in Form von vier Tabellen mit Kommentar, in denen die Aussprache, Grammatik, Wortschatz und die Gegenüberstellung der Sprachvarietäten und der Standardsprache dargestellt werden. Den Schlussfolgerungen folgt ein Appendix mit den Übersetzungsstrategien zu kulturspezifischen Wörtern im audiovisuellen Übersetzen von Jan Pedersen und ein Literaturverzeichnis, mit dem die Publikation schließt.

Die Einleitung bildet eine theoretische Basis für die in den jeweiligen Kapiteln präsentierten Analysen. Einleitend greift die Autorin die Thematik der Untertitelung als eine Art des audiovisuellen Übersetzens auf. Dabei kommt sie auf die wichtigsten Merkmale dieser Übersetzungsart zu sprechen. Daran anschließend werden sprachliche Varietäten in Filmen als eine Herausforderung für den Übersetzer dargestellt. Schließlich wird das Konzept der Heteroglossie von Michail Bachtin erörtert, auf das Ellender ihre Ausführungen stützt. 